\title{
Optical coherence tomography multichannel probe design for speckle reduction (Withdrawal Notice)
}

Dongyao Cui, En Bo, Yuemei Luo, Xinyu Liu, Xianghong Wang, et al.

Dongyao Cui, En Bo, Yuemei Luo, Xinyu Liu, Xianghong Wang, Si Chen, Xiaojun Yu, Shi Chen, Ping Shum, Linbo Liu, "Optical coherence tomography multichannel probe design for speckle reduction (Withdrawal Notice)," Proc. SPIE 10340, International Conference on Biophotonics V, 103401E (29 April 2017); doi: 10.1117/12.2271547

SPIE. Event: International Conference on Biophotonics V, 2017, Perth, Australia 


\section{Optical coherence tomography multichannel probe design for speckle reduction (Withdrawal Notice)}

Proc. SPIE 10340, 103401E (2017)

Online Publication Date: 29 April 2017

Withdrawn from Publication: 9 May 2017

Conference Date: 30 April-1May 2017

Conference Location: Perth, Australia

Conference Title: International Conference on Biophotonics $\mathrm{V}$

Conference Chairs: David D. Sampson, Dennis L. Matthews, Jürgen Popp, Halina Rubinsztein-Dunlop, Brian C. Wilson

Dongyao Cui, En Bo, Yuemei Luo, Xinyu Liu, Xianghong Wang, Si Chen, Xiaojun Yu, Shi Chen, Ping Shum, and Linbo Liu

Nanyang Technological Univ. (Singapore)

This paper has been withdrawn by the publisher at the request of the authors. 